\title{
Optimization of Backscattered Electron (BSE) Detector for Variable Pressure SEM with Monte Carlo method
}

\author{
Z. Hafsi ${ }^{1,2 *}$, L.Djemoui ${ }^{3}$ O. Mansour ${ }^{2}$, A. Kadoun ${ }^{2}$ \\ ${ }^{1}$ Département Automatique et électromécanique, Facuté science et technologie, université de Ghardaia, Algeria \\ ${ }^{2}$ Laboratoire de Microscopie, Microanalyse de la matière et Spectroscopie Moléculaire, Faculté des Sciences Exactes, Djilali \\ Liabès university, 22000 Sidi Bel-Abbès, Algeria. \\ ${ }^{3}$ Laboratory of Materials, Energy Systems Technology and Environment "MESTEL”, Faculté des sciences et technologie, \\ Université de Ghardaia, Algeria
}

*Email: hafsidoc@yahoo.com

\begin{abstract}
This work deals with The study of the energy and angular distributions of backscattered electrons (BSE) detected under vacuum and with pressure, which contribute to determining the appropriate place to put the BSE detector, we examine vacuum and air gas, the diameters of detector, we discuss its influence on the energy and angular distribution of BSE in the sample by Monte Carlo simulation to find the optimum experimental parameters needed for BSE imaging in VPSEM. For this aim, we have chosen: $\mathrm{SiO} 2$ as dielectric material and carbon for his lower atomic numberin our results under vacuum shows that the limited geometry of the detector (detector radius) causes a great reduction of the BSE signal, The analyses demonstrate the location of a BSE detector relative to a sample surface will have a strong influence on its collection efficiency.
\end{abstract}

Keywords: V-PSEM, Backscattered electron (BSE), Energy and angular distributions, MC simulations.

\section{INTRODUCTION}

Several authors have studies theoretically and experimental about the properties of backscattered electrons (BSE) and their depend on their coefficient (BSE coefficient $\eta$ ), angular distributions and their energy distributions. [1-20].

The signal of the backscattered electrons (BSE) can be detected by the same means as in high-vacuum mode conditions at scanning electron microscopy (SEM). Improvements were made to the BSE detector first by Robinson [21] Then, backscattered electron imaging reached its peak following the use of scintillator type sensors at environmental SEM (Danilatos, 1985) [22]. Unfortunately, so far this has remained only as a prototype laboratory (Danilatos) [23]. In general, the backscattered electron detectors are based on: the material used for detection, the state of the characterized material and the type of gas used for imaging and its pressure in addition to the detector polarization and working distance.

In this we examine the influence of producer gas on the energy and therefore the angular distributions of backscattered electrons from carbon and $\mathrm{SiO} 2$ by Monte Carlo simulation to seek out the optimum experimental parameters needed for BSE imaging in Variable Pressure SEM.

\section{SIMULATION DETAILS}

We used the Monte Carlo simulation that we used in Hafsi and al [24] where These modeling we built base on the two models joint together david joy[25] and Mansour and al [26], , licenses all the exploratory factors (gas composition, weight; working remove, the speeding up voltage and the test characteristics) to be chosen and tracks each electron from the point where it 
enters the moo vacuum locale until it comes to the test surface, permitting the point by point bar profile to be decided.

Moreover, in our calculations, the electron takes after a Gaussian dissemination earlier because it passage in gas. $10^{6}$ electrons trajectory was done. We consider air gas. The simulation conditions are: an accelerating voltage of 5 and $20 \mathrm{kV}$ and a working distance of $2 \mathrm{~mm}$. The BSE detector is assumed to be annular but split intotwo halves quadrants about the incident beam- the outer diameter of the detector is $\mathrm{R}=5 \mathrm{~mm}$.

For the BSE coefficient ( $\eta$ ) and the spatial distributions, our models predict the energies and exit angles of the backscattered electrons. Essentially, the procedure we followed was to model $10^{6}$ electrons at 5 and $20 \mathrm{keV}$ for each target.

We then looked at the trajectory of each electron that wa s backscattered, calculated its energy, and its angle betw een that trajectory and the normal surface.

Then we set up counting intervals corresponding to 100 equal divisions of the interval $[0,1]$ (where 0 to 1 is the range of possible values, reduced energy $\left(\mathrm{W}=\mathrm{E}_{\mathrm{bse}} / \mathrm{E}_{0}\right)$. finally,for each backscattered electron, we simply incremented the count in each interval corresponding to the value $\mathrm{W}$.At this stage, plotting the number of electrons in the interval corresponding to $0.01<\mathrm{W}<0.1$ at the coordinate $\mathrm{x} 0.1$, and so on, would give a curve representing BSE vs W. However, we need a graph the distribution of energy $d \eta / d E$ vs $W$ to compare with experience.

The main characteristics of the energy distribution are the relative stability of its forme, the dominance of electrons having suffered significant energy losses, he absence of an outsized group of electrons having undergone elastic diffusions, and the slow increase of average energy with the increase in the atomic number. For the angular distribution of the BSE $\mathrm{d} \eta / \mathrm{d} \theta$, the same procedure described above will be followed.

\section{RESULTS AND DISCUSSION}

\subsection{Energy Distribution}

Now, in order to validate our simulation model regarding the energy distributions of BSE, we propose in this paragraph to compare our results to those ofpublished research, whether theoretical or experimental. Unfortunately, carbon data, are only available in the literature for large energies, more rarely for $5 \mathrm{keV}$ energies. For the $\mathrm{SiO} 2$ the results are almost nonexistent, except at $0.5 \mathrm{keV}$ by Dapor [12]. To do this, we have chosen the Aluminum, where the results are abundant, and which allow this comparison

Figure 1(a, b) represents a comparison between our simulation results (MC) and the experimental results of Matsukawa [2] and Darlington [3] at 20keV, which are standardized, and Yadav [13] for the Aluminum at $5 \mathrm{keV}$. It is noted that for Staub [6] the curves represent its theoretical formula (see below).
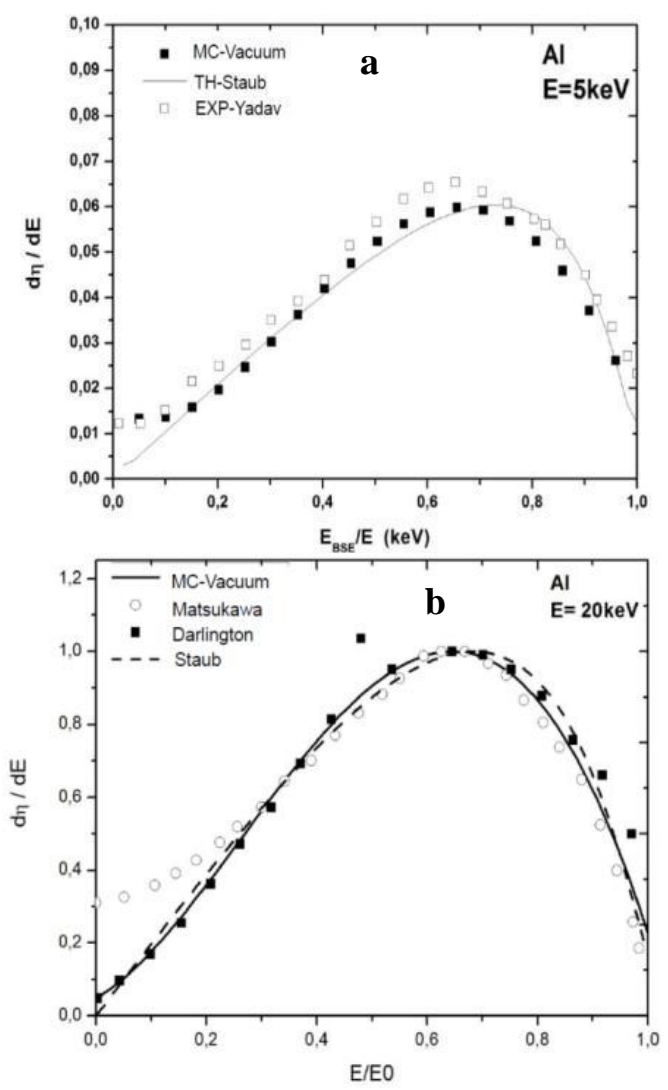

Figure 1 a)BSE Energy Distributions for Aluminum $5 \mathrm{keV}$ at Vacuum b) BSE standard energy distributions for $20 \mathrm{keV}$ aluminum at vacuum

In these spectra from different experiments, it is found that there is a slight variation in energy that could be due to the uncertainties of calibration of the experiments[14]. There are large variations among different experiments in the lower parts of the spectra. These differences are mainly due to the resolution of the detector in this energy range [1]. Given the variations between experiments, it can be said that the spectragenerated by our program are well in the average of the experimental measurements.

We compared our results with the theoretical model of Staub, which predicts the energy distributions of backscattered electrons. The energy of electron spectra backscattered for a zero angle of incidence during this model is given by[6]: 


$$
\begin{aligned}
g(E / E) & =-\frac{\partial \eta\left(E_{B} E_{0}\right)}{B \quad 0} \\
\eta\left(E_{B} / E_{0}\right) & =\operatorname{S} \cdot \exp \left[-\left(\frac{\partial E_{B}}{1-\gamma\left(E_{B} / E_{0}\right)^{\alpha}}\right) \mathrm{p}\right]
\end{aligned}
$$

$\mathrm{S}, \mathrm{K}, \mathrm{p}, \gamma, \alpha$ independent parameters $\mathrm{K}=70 \operatorname{Ln} B^{4}$ ,p=0.27, $\gamma=1$-ep $\left(-6 \operatorname{Ln} B^{-3 / 2}\right), \alpha=2.0$ et $\mathrm{S}$ est la normalised fonction given by:

$$
\begin{aligned}
& \mathrm{S}=\mathrm{B}_{0} \exp \left[-(\mathrm{k})^{\mathrm{p}}\right] \\
& \mathrm{B}_{0}=\beta\left\{1-\exp \left[\left(-6.6 \times 10^{-3}\right) \times \beta^{-5 / 2} Z\right]\right\}
\end{aligned}
$$

Où $\mathrm{B}_{0}$ the coefficient of backscattered electron with normal incidence

$$
\beta=0.40+0.065 \ln \mathrm{E}_{0}
$$

It is noted that the theory not precise the data in the region corresponding to $\mathrm{W} \leq 0,1$. This may be due to thefact that in this region most of the electrons detected are a mixture of secondary electrons the with low-energy $(\mathrm{E} \leq 50 \mathrm{eV})$ and the backscattered electrons with energies $\leq 1000 \mathrm{eV}$ (Figure 2).The secondary electrons are generally produced by the primary electron beam and by backscattered electrons. We also interpret it by consider some of the scattered electrons can obtain higher kinetic energy under the influence of the load space force resulting from the collisions. Figure 1 also overstates experimental data in the peak region between

0.7 and 0.9 ; this is due to the low elastic scattering of incidence electrons in low atomic number.

It is also noted that the energy spectrum (dE) for $\mathrm{Al}, \mathrm{C}$ and $\mathrm{SiO}_{2}$ depend on the $\mathrm{Z}$ target, especially the high atomic number elements have a distribution of up to a peak of about 1 in relation to low atomic number that have peaks at lower values. This trend can be explained qualitatively by noting that high $\mathrm{Z}$ with a high probability of elastic scattering can product more backscattered electrons near to incident energy.

Figure 2 shows the difference between the curve of electrons from the surface detected under vacuum and air pressure at $500 \mathrm{~Pa}$ for a $5 \mathrm{~mm}$ detector radius. Further on, we will focus on the energies of the carbon- and $\mathrm{SiO} 2-$ based electrons reaching the detector.

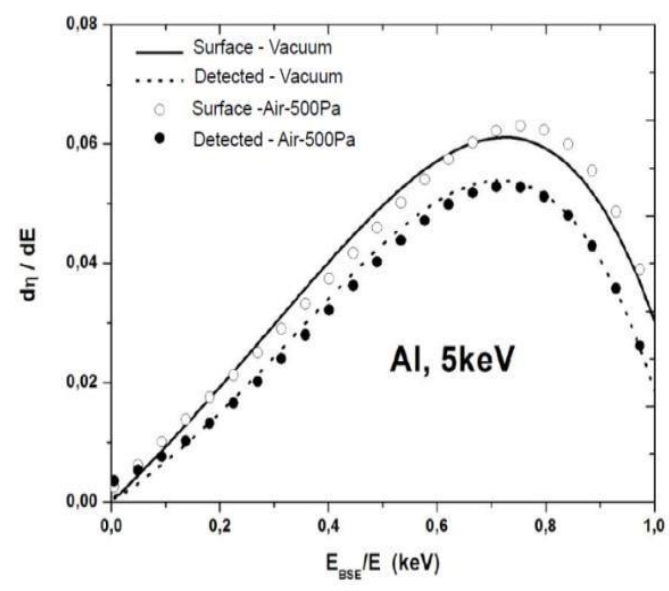

Figure 2 The energy distributions ofBSE at the surface and detected for the $5 \mathrm{keV}$ aluminum under vacuum and air pressure $\mathrm{P}=500 \mathrm{~Pa}$

The distributions of plotting energies for $\mathrm{SiO} 2$ and carbon at 5 and $20 \mathrm{keV}$ in relation to air pressure, which is the most unfavorable case for electron diffusion, are shown in figure $1(\mathrm{a}, \mathrm{b})$. As mentioned above, it can be noted that the regions under the energy spectrum of the two targets represent their backscatter coefficient values $\eta$. At These regions the backscattered electrons increases with the atomic number. Also, as expected, there is a large reduction in the amplitude between the energies of the BSE on the aluminum surface and the detector (Figure 3). This reduction is actually due to the limited geometry of the detector.

Under air pressure at $500 \mathrm{~Pa}$, the surface spectrum increases and shifts to the right towards energies above $0.6 \mathrm{keV}$ (Figure 3). That is usually due to the inclination of the skirt radius. Incident electrons that belong to the skirt radius are slightly inclined to the incident scattering, giving rise to a situation similar to the one where the sample is a little inclined.

In addition, the electrons of the skirt, which have undergone significant angular deviations, move with a small path in the target before being backscattered and lose less energy than those with weak deviations. Generally, the BSE coefficient increases to an oblique incidence.

This also appears to take into account the increase in the energy spectrum of BSE from the sample surface with the increase in pressure.

On the other hand, the spectrum decreases slightly between 0.2 and $0.6 \mathrm{keV}$ (Figure3). It is known that at low energy, the gas cross section increases and the mean free path decreases, increasing collisions and energy losses of BSE before colliding with the material. Once 
in the latter, they still lose energy, reflecting this slight decrease in spectra between 0.2 and $0.6 \mathrm{keV}$.

For both materials, we calculated the most probable detected energy of BSE Table1. These energies increase with the atomic number of each material, and decrease with the pressure $(250 \mathrm{~Pa})$ of low-energy air $(5 \mathrm{keV})$.

We note, $4 \%$ loss for the $\mathrm{SiO} 2,5 \%$ loss for carbon. At $20 \mathrm{keV}$, the effect of the pressure has no effect on the energy variation. This is due to the low fraction released at $250 \mathrm{~Pa}$. For $5 \mathrm{keV}$, the probability of collision is high, and BSEs experience gas collisions by losing some of their energy, which explains the most likely reduction in energy detected.

Table 1. The most probable BSE energy Ep for different pressure and for the two materials $\mathrm{C}, \mathrm{SiO} 2$, to $\mathrm{E}=5 \mathrm{keV}, 20 \mathrm{keV}$

\begin{tabular}{|lccc|}
\hline \multicolumn{4}{|c|}{ Most I probable detected energy (Ep), under air } \\
pressure \\
\hline & $5 \mathrm{keV}$ & $20 \mathrm{keV}$ \\
Vacuum & 3.35 & $\mathrm{SiO}_{2}$ & 13 \\
$250 \mathrm{~Pa}$ & 3.20 & & 13 \\
\hline & & Carbone & \\
Vide & 2.95 & & 12 \\
$250 \mathrm{~Pa}$ & 2.80 & & 12 \\
\hline
\end{tabular}

\subsection{Angular distributions}

Other interesting BSE properties are their angular distribution relative to the direction of electron beam incidence.

The angular distribution of the backscattered electrons when emerging from the surface of the sample covers a solid angle of 180 degrees, but the actual amount detected on the signal depends on the geometry and the detector collection mechanism. In this section, we study the angular distribution of BSE from the carbon surface and $\mathrm{SiO} 2$ between 0 and $90^{\circ}$ due to signal symmetry. The results are plotted in figure(3.4) for different pressures and energies.
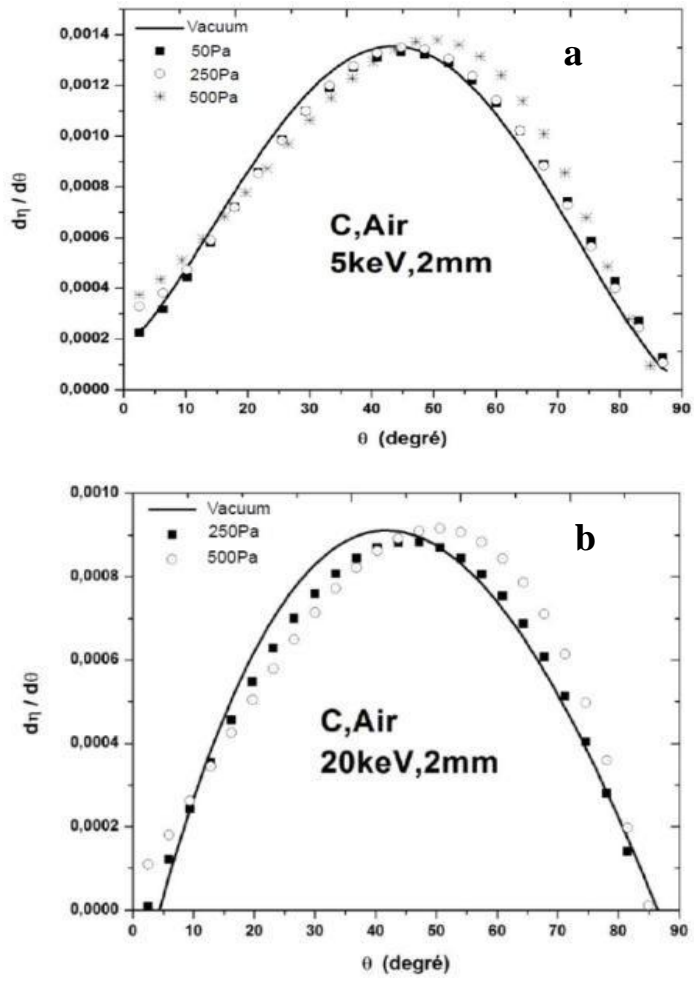

Figure 3 Angular distribution of BSE at carbon surface for different air pressure at a) $5 \mathrm{keV} \mathrm{b}) 20 \mathrm{keV}$
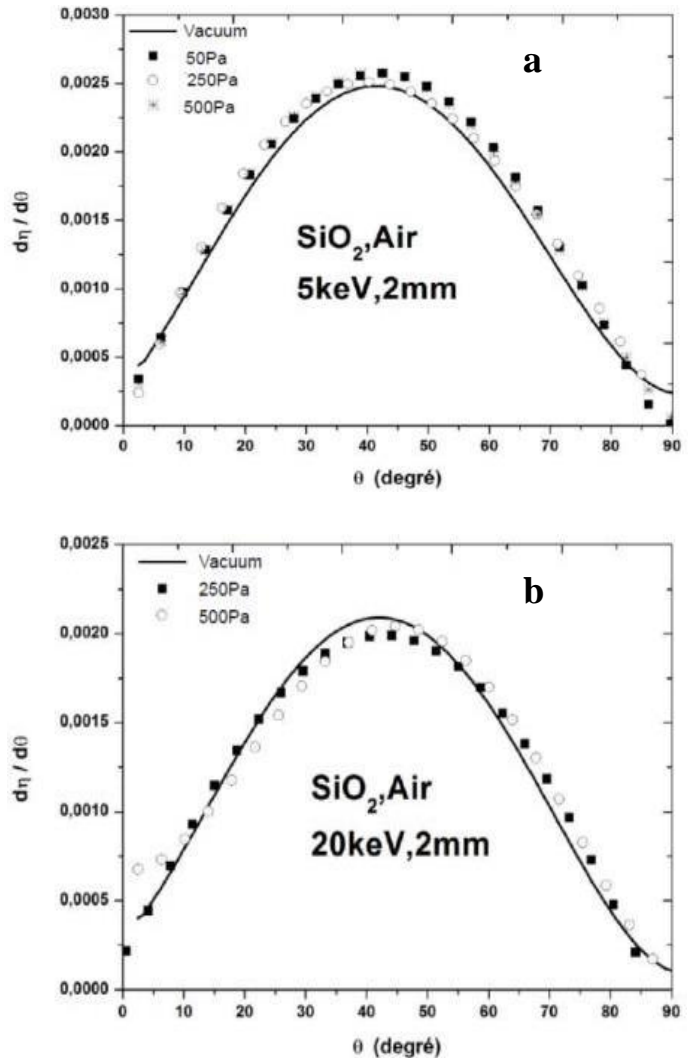

Figure 4 Angular distribution of BSE at the surface of $\mathrm{SiO} 2$ for different air pressure at a) $5 \mathrm{keV}$ b) $20 \mathrm{keV}$ 
For carbon, the pressure variation is more pronounced on the detected angles than for $\mathrm{SiO} 2$. A shift in the spectrum to the upper angles $\left(>45^{\circ}\right)$ and lower angles to $<10^{\circ}$ is observed with increasing pressure. This variation is always related to the radius of skirt and its impact on the variation of BSE by atomic number. Under gas pressure, the scattered electrons (skirt) hit thetarget with non-zero initial angles and quickly come out as backscattered electrons without losing much energy. These initial angles are in addition to the number of times the azimutal angles are calculated during the electron journey for each material. The farther the electron is from the point of impact, the greater theangle of escape, especially for low density materials

For carbon and $\mathrm{SiO} 2$, we calculated the most likelyangle detected Table 2 below. These angles decrease at $5 \mathrm{keV}$, and increase slightly at $20 \mathrm{keV}$ with the increasein the atomic number. This variation in vacuum is due tothe variation in density of the two materials, atomicnumber and interaction volume.

At $5 \mathrm{keV}$, each time $\mathrm{Z}$ increases, the density increases, the interaction volume narrows and the angles decrease. At $20 \mathrm{keV}$, the angles increase because the interaction volume is increased by about 10 times compared to $5 \mathrm{keV}$. Depending on the air pressure at $(5 \mathrm{keV}, 2 \%$ increase is recorded from the most probable angle for carbon and $6 \%$ at $20 \mathrm{keV}$, for $\mathrm{SiO} 2$ the pressure of $250 \mathrm{~Pa}$ or the energy no noticeable effect on the variation of the detected angles.

Table 2. The detected angle of the BSE most probably for different pressure and for different materials $\mathrm{C}, \mathrm{SiO} 2$ to $\mathrm{E}=5 \mathrm{keV}, 20 \mathrm{keV}$

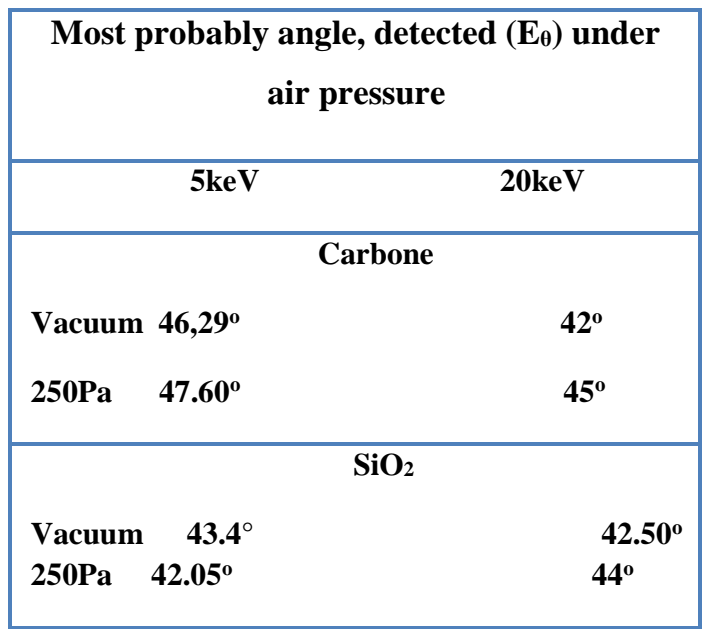

\section{CONCLUSION}

Study of energy and angular distributions of the BSE detected at vacuum shows that the limited geometry of the detector (detector radius) leads to a large reduction of the BSE signal where the most likely energies range from $0.6 \mathrm{E} 0$ to $0.67 \mathrm{E} 0$. Under air pressure, the effect of the skirt results in a shift of the spectra towards the energies and upper angles, especially for carbon.Angular distribution inspection shows that the maximum number of backscattered electrons is emitted from materials at 5 and $20 \mathrm{keV}$ at $<250 \mathrm{~Pa}$ pressures, along the normal surface at angles that vary between $42^{\circ}$ and $46^{\circ}$ which are the most probable angles. If the detector is placed at higher or lower values, the backscattered electrons decrease. At the furthermost angles, just up on the surface, the location of BSE detector relative to a sample surface will have a strong influence on its collection efficiency.

\section{ACKNOWLEDGMENTS}

I thank the following individuals professor O.MANSUR, and A. Kadoun for their expertise and assistance throughout all aspects of our study and for their help in writing the manuscript.

\section{REFERENCES}

[1] Bishop H E, : Some electron backscattering measurements for solid targets Proc. 4th Int. Conf. on X-ray Optics and X-ray Microanalysis ed R Castaing et al (Paris: Hermann),153-8(1965) . Doi :

https://doi.org//10.1063/1.331005

[2]T. Matsukawa, R. Shimizu and H .Hashimoto, Measurements of the energy distribution of backscattered kilovolt electrons with a spherical retarding-field energy analyser J. Phys. D: Appl. Phys., 7 695-702(1974). DOI: https://doi.org /10.1007/978-3540-38967-5_4

[3] E .H .Darlington, Backscattering of 10-100 keV electrons from thick targets J. Phys. D: Appl. Phys. 8 85-93(1975). DOI: https://doi.org/10.1088/0022$3727 / 8 / 1 / 016$

[4] M. R. Sogard ,Energy spectra and angular distributions of charged particles backscattered from solid targets J. Appl. Phys. 53, R15 10.1063/1.331005(1982). DOI: https://doi.org/ 10.1088/0022-3727/41/5/055505

[5] M. del Giorgio and J. Trincavelli ,Backscattering of 10-35 keV Electrons from Thick Targets at Normal 
Incidence,X-RAY SPECTROMETRY, VOL. 18,229234(1989).

[6] P. F. J, Staub , Phys. D 27,1533(1994) .

[7] M. SPRANCK, M. KÄSSENS, L. REIMER, Influence of the Angular Distribution of Backscattered Electrons on Signals at Different Take-Off Angles in Low-Voltage Scanning Electron Microscopy (LVSEM),SCANNING Vol. (1995) 17, 97-105.DOI:

https://doi.org/10.1002/sca.4950170206

[8] G. Patrick Watson, Measurement of the backscatter coefficient using resist response curves for $20-100 \mathrm{keV}$ electron beam lithography on Si J. Vac. Sci. Technol. B $14,4277,10.1116 / 1.588590$.

[9] M .Dapor and A. Miotello Backscattering of electrons from selected oxides: $\mathrm{MgO}, \mathrm{SiO}_{2}$, and $\mathrm{Al}_{2} \mathrm{O}_{3}$, Eur. Phys. J. AP 5,143-148(1999). Doi: https://doi.org 10.1051/epjap:1999122

[10] R.K .Yadav, and Shanker, Energy and angular distributions of backscattered electrons from the collision of 8-keV electrons with a thick tungsten target. PHYSICAL REVIEW A 70,052901(2004) .Doi: https://doi.org/10.1103/PhysRevA.70.052901

[11] M.Dapor, A comparative study of electron and positron penetration in silicon dioxide, J. Electron Spectrosc. Related Phenomena 151, 182192(2006).Doi:

http://dx.doi.org/10.1016/j.elspec.2005.12.005

[12] M,Dapor, Monte Carlo computations of the electron backscattering coefficient for bulk targets and surface thin films, Surf. Interface Anal 40714717(2007) .Doi: https://doi.org/10.1002/sia.2637

[13] R.K .Yadav, and Shanker, Energy and angular distributions of backscattered electrons from collisions of $5 \mathrm{keV}$ electrons with thick $\mathrm{Al}, \mathrm{Ti}, \mathrm{Ag}, \mathrm{W}$ and $\mathrm{Pt}$ targets, journal physics 517-528(2007) .Doi: https://doi.org/ 10.1007/s12043-007-0055-Z

[14] E S M Ali and D W O Rogers, Benchmarking EGSnrc in the kilovoltage energy range against experimental measurements of charged particle backscatter coefficients Phys. Med. Biol. 53,152743(2008) .Doi: https://doi.org/ 10.1088/00319155/53/6/002

[15] M. Dapor, N. Bazzanella , L. Toniutti , A.Miotello, S. Gialanellaal., Nucl. Instrum. Methods Phys.269, 1672-1674(2011).

[16] J. Cazaux, Electron back-scattering coefficient below 5keV: Analytical expressions and surface-barrier effects, J. Appl. Phys,112, 084905.(2012).Doi: https://doi.org/10.1063/1.4759367

[17] T. Kowoll, E. Müller, S.Fritsch-Decker, S. Hettler, H. Störmer, C. Weiss, and D. Gerthsen, Contrast of
Backscattered Electron SEM Images of Nanoparticles on Substrates with Complex Structure, Hindawi Scanning 12 pages( 2017). Doi: https://doi.org/ $10.1155 / 2017 / 4907457$

[18] A. M. D. Assa'd, Monte Carlo calculation of the backscattering coefficient of thin films of low on high atomic number materials and the reverse as a function of the incident electron energy and film thickness Applied Physics A 124:699(2018). Doi: https://doi.org/ 10.1007/s00339-018-2073-8

[19] S. Kahane, Backscattered Electrons Spectra and Angular Distributions: Simulations with EGS5 , EJERS, European Journal of Engineering Research and Science Vol. 3, No. 10, October 2018 .

[20] A.Hussain , L. Yang , S.Mao b,*., B. Da c, K. T"ok'esi , Z.J. Ding , Determination of electron backscattering coefficient of beryllium by a highprecision Monte Carlo simulation Nuclear Materials and Energy 26 100862(2021) . Doi:https://doi.org/ 10.1016/j.nme.2020.100862

[21] V.N.E, Robinson, The elimination of charging artifacts in the scanning electron microscope. J. Phys. E: Sci. Instr. 8638-640(1975).

[22] G. D. Danilatos, Design and construction of an atmospheric or environmental SEM (part 3). Scanning 7,26-42(1985).

[23]G. D. Danilatos , Backscattered electron detection in environmental SEM. Journal of Microscopy 245, 171-185(2012). Doi: https://doi.org/10.1111/j.13652818.2011.03559.x

[24] Z. Hafsi, O. Mansour , A. Kadoun , L. Khouchaf, C. Mathieu ,Gas effect on the emission and detection of the backscattered electrons in a VP-SEM at low energy, Ultramicroscopy 184 (2018) 17-23.Doi: Doi: https://doi.org/10.1016/j.ultramic.2017.08.002

[25] D.C. Joy, An introduction to Monte Carlo simulation, Scan Microsc. 5, 329-337(1991).

[26] O. Mansour, K. Aidaoui, A. Kadoun, L. Khouchaf, C. Mathieu, Monte Carlo sim- ulation of the electron beam scattering under gas mixtures environment in an HPSEM at low energy, Vacuum 84 ,458-463(2010). 